\title{
Newer Updates in Psychiatry: Focus on Vortioxetine
}

Imon Paul

\begin{abstract}
Vortioxetine is the new antidepressant on the block which has been described to have a novel mechanism of action-it directly works on multiple receptors of the neurotransmitter serotonin and it also inhibits serotonin reuptake. It has been shown to have promising antidepressant, antianxiety and pro-cognitive action especially during treatment of major depression. This article gives a brief overview of vortioxetine-its clinical use, pharmacological profile, and safety and tolerability. Most of the studies till date have found vortioxetine to be clinically efficacious, and it also improved the cognitive profile in patients with depression. It has a favorable adverse effect profile- it has been shown to be tolerated well by most subjects in clinical trials where nausea was the most frequently reported side effect, and it also showed the added advantage of comparatively less incidence of unwarranted sexual adverse effects.
\end{abstract}

Keywords: Adverse events, Major depressive disorder, Vortioxetine.

How to cite this article: Paul I. Newer Updates in Psychiatry: Focus on Vortioxetine. Ind J Priv Psychiatry 2018;12(2):62-65.

Source of support: Nil

Conflict of interest: None

\section{INTRODUCTION}

In the current scenario, major depressive disorder (MDD) is widely prevalent with both emotional and physical manifestations. Depression has wide-ranging negative effects on the sufferer's mental and physical well being, and it leads to persistent sadness and anhedonia. The global burden of depression can be apprehended by the fact that according to estimates by the World Health Organization (WHO) depression is slated to become the second major reason for disability in the world by the year $2020 .{ }^{1}$ Despite the widespread prevalence, major depression is often unrecognized and untreated, leading to tragic consequences, like impaired quality of life and suicide. The main treatment options available currently for depressive disorders include both pharmacotherapies as well as psychotherapy. Treatment aims to bring about

\footnotetext{
Associate Professor

Department of Psychiatry, IQ City Medical College, Sovapur, Bijra Road, Jemua Durgapur, West Bengal, India

Corresponding Author: Imon Paul, Associate Professor, Department of Psychiatry, IQ City Medical College, Sovapur, Bijra Road, Jemua Durgapur, West Bengal, India, e-mail: dr.imonpaul@gmail.com
}

improvement in mood and depressive cognition and ultimately, the quality of life. Though there are many treatment options available for the management of MDD, concerns regarding efficacy and tolerability have been persistent, particularly in respect to sexual dysfunction, cognitive impairment, and causation of weight gain, which can lead to poor treatment compliance. Hence, the search for the "holy grail" of antidepressant medication continues and the advent of vortioxetine with a purportedly novel multimodal action and safety profile has generated strong interest.

\section{VORTIOXETINE: A NEW AGENT FOR MDD}

Vortioxetine was approved by the United States Food and Drug Administration (USFDA) as a therapeutic option in the management of adult patients suffering from MDD in 2013. ${ }^{2}$

\section{Pharmacology}

Vortioxetine has a chemical formula of 1-[2-(2,4-dimethylphenylsulfanyl)-phenyl]-piperazine; Lu AA21004). It is a new agent with purported multimodal activity. It works on multiple receptors of the neurotransmitter serotonin (antagonist at 5-HT3, 5-HT7, 5-HT1D receptor, a partial agonist at 5-HT1B receptor and agonist at 5-HT1A receptor) and also inhibits the transporter of serotonin (SERT). ${ }^{3-6}$ Vortioxetine has a bioavailability of around $75 \%$ after oral administration and bio availability remains unchanged by food intake. Peak plasma concentration $\left(\mathrm{T}_{\max }\right)$ is achieved in about 7 to 8 hours and the half-life of Vortioxetine $\left(t_{1 / 2}\right)$ is approximately 57 hours. The steadystate concentration is reached in less than 14 days. Vortioxetine is highly bound to plasma proteins (around 96\%). It is metabolized in an extensive manner, mainly by the process of oxidation followed by glucuronic acid conjugation. The P-450 enzymes involved in metabolizing Vortioxetine are CYP2D6 CYP3A4/5, CYP2C9, CYP2C19, CYP2A6, CYP2C8, and CYP2B6. ${ }^{7}$ CYP2D6 is the chief enzyme which catalyzes the breakdown of the active molecule to its pharmacologically inactive carboxylic acid metabolite. Hence there is a high possibility of drug interactions when vortioxetine is administered with CYP2D6 inhibitors and inducers and adjustment in dosage may be necessary for such a scenario. Vortioxetine does not inhibit or induce the P-450 enzymes significantly. ${ }^{8}$ Vortioxetine is 
currently available in the dose strengths of 5, 10, 15 and $20 \mathrm{mg}$ tablets. Vortioxetine can be initiated at a dose of $10 \mathrm{mg} /$ day, and gradually hiked to $20 \mathrm{mg}$ daily, according to tolerability. As vortioxetine has a long half-life, there is less likelihood of discontinuation symptoms if it is discontinued abruptly; but it is preferable that higher doses be gradually tapered before full discontinuation. ${ }^{9}$

\section{Vortioxetine-How Effective is it?}

Multiple short-term, as well as long-term trials, were done to assess the efficacy of vortioxetine in the management of clinical depression. Out of twelve short-term clinical trials, ${ }^{10-21}$ eight trials showed favorable results with vortioxetine (seven of these trials found vortioxetine to be superior to placebo and one trial showed favorable outcome with vortioxetine which was compared to agomelatine). Three trials could not find any significant advantage of vortioxetine over placebo in the treatment of depression. One study compared vortioxetine with duloxetine, but neither medication was superior to placebo. Subjects generally showed a response two weeks after initiating vortioxetine, and the maximum improvement was seen after a month of treatment. ${ }^{22}$ As clinical depression often runs a chronic course and relapses are common, long-term efficacy studies are also essential to establish the role of a therapeutic agent in the management of depression. A long-term study which compared vortioxetine with placebo reported that patients with depression on vortioxetine had relapse rates of $13 \%$ which was significantly less than the relapse rate of $26 \%$ seen in the placebo group. ${ }^{23}$ Another study by Baldwin et al. ${ }^{10}$ was extended to observe the effects of vortioxetine over time. ${ }^{24}$ At the conclusion of the second study which lasted for 52 weeks, the percentage of responders to vortioxetine increased from 63 to $94 \%$ and the remission rate of depression improved to $83 \%$ compared to $42 \%$ in the initial study. ${ }^{24}$

\section{Vortioxetine and Cognition}

In a small trial comparing vortioxetine with mirtazapine, vortioxetine did not negatively affect driving, psychomotor or cognitive performance with acute or chronic administration while mirtazapine, the active comparator, showed cognitive and psychomotor impairment after single dose administration. These results suggested that vortioxetine does not adversely impact cognition. ${ }^{25}$ Several clinical studies were undertaken to demonstrate the extent of vortioxetine's independent pro-cognitive effects. The common neuropsychological tests used for evaluation of cognitive status include the rey auditory verbal learning test (RAVLT) and the digit symbol substitution test (DSST). Vortioxetine was superior when compared to placebo in terms of better performance in the above mentioned cognitive neuropsychological tests in elderly depressed patients. ${ }^{11}$ In one clinical trial which specifically evaluated how vortioxetine affects the cognitive functioning in depressed persons, it was found that vortioxetine showed significant improvement in cognition as measured by RAVLT and DSST versus placebo. ${ }^{12}$ Mahableshwarkar et al. conducted a randomized study specifically assessing the role of vortioxetine on cognition in patients with depression who also reported cognitive impairment. ${ }^{26}$ Vortioxetine was found to be better than placebo when the change from baseline of DSST score was considered as the primary endpoint, as well as perceived deficit questionnaire scores. It has also been suggested that vortioxetine has an independent effect on cognitive performance and it is not merely because of improvement in the depressive symptomatology as the procognitive effect of vortioxetine is maintained even when corrected for the effect on depressive symptoms. ${ }^{12}$

\section{Vortioxetine: How Well is it Tolerated?}

The evaluation of safety data from clinical trials indicates that vortioxetine is tolerated well by most subjects. As the molecule has a prolonged half-life, there is less likelihood of occurrence of discontinuation symptoms if it is stopped abruptly. The analysis of clinical trials of vortioxetine revealed that most of the medication-related adverse events reported by patients on the therapeutic dosage of vortioxetine were of mild or moderate intensity. ${ }^{27}$ The most frequently reported side effect was nausea, and it appeared to be dose-related. ${ }^{27,28}$ Nausea was usually transient, lasting for 10 to 16 days on an average and commonly occurred in the initial phase of treatment. The occurrence of sleep-related adverse effects in patients on vortioxetine ranged between 2.0 to $5.1 \%$ which was comparable to the rates of sleep-related disturbances seen with placebo. ${ }^{27}$ The occurrence of spontaneously self-disclosed sexual dysfunction was low in randomized as well as openlabel trials of vortioxetine. ${ }^{28}$ This is especially significant as sexual dysfunction is often under-reported though it is a very common side effect associated with antidepressant medication that can often lead to nonadherence to treatment. In a large, double-blind clinical trial lasting for 8 weeks, 447 patients suffering from MDD and with sexual adverse effects related to SSRI therapy were randomized to switch to one of the two agents-either vortioxetine or escitalopram. ${ }^{29}$ The primary outcome measure at the end of the trial was a change from baseline in the changes in sexual functioning questionnaire shortform (CSFQ-14) score. The patients on vortioxetine had significantly greater improvement in CSFQ-14 score when compared to the patients on escitalopram. Secondary endpoints assessing clinical efficacy were comparable 
between the two groups. This study showed potential for vortioxetine to reverse sexual dysfunction caused by SSRI therapy while maintaining clinical efficacy.

\section{DISCUSSION}

Vortioxetine is an emergent therapeutic agent which is effective in managing acutely depressed patients as well as for preventing relapse of depressive symptomatology in adult patients with MDD. Since it acts at multiple receptors, it also shows antianxiety and procognitive effects during the treatment. The majority of studies done till date have shown vortioxetine to be efficacious and well tolerated. Nausea has been reported as the most frequently occurring side effect. Another advantage of vortioxetine is that the occurrence of sexual adverse effects is relatively less and it does not cause any significant change in weight, factors which are likely to lead to better adherence to treatment. Since MDD has widespread prevalence and leads to significant clinical and economic burden globally, the emergence of vortioxetine looks promising as it also has the potential of mitigating the cognitive impairment associated with depression in adult patients and may signal future applications of this agent in other mental disorders in the domain of cognitive dysfunction. However, further studies comparing vortioxetine with established agents are required to comprehensively establish the effectiveness of vortioxetine and its future use in clinical psychiatry.

\section{REFERENCES}

1. World Health Organization and Department of Mental Health and Substance Abuse. Victorian Health Promotion Foundation. University of Melbourne. Promoting mental health: concepts, emerging evidence, practice: summary report. Geneva, Switzerland: World Health Organization; 2004. Available from: http://www.who.int/mental_health/evidence/en/ promoting_mhh.pdf. Accessed July 22, 2015.

2. US Food and Drug Administration. FDA approves new drug to treat major depressivedisorder. Press release. September 30, 2013. Updated October 10, 2013.www.fda.gov/NewsEvents/ Newsroom/PressAnnouncements/ucm370416.htm. Accessed October 23, 2013.

3. Stahl SM. Stahl's Essential Psychopharmacology:Neuroscien tific Basis and Practical Application. 4th ed. New York, New York: Cambridge University Press; 2013.

4. Celada P, Bortolozzi A, Artigas F. Serotonin 5-HT 1A receptors as targets for agents to treat psychiatric disorders: rationale and current status of research. CNS drugs. 2013 Sep 1;27(9):703-716.

5. Owens MJ, Nemeroff CB. Role of serotonin in the pathophysiology of depression: focus on the serotonin transporter. Clinical chemistry. 1994 Feb 1;40(2):288-295.

6. Bang-Andersen B, Ruhland T, Jørgensen M, Smith G, Frederiksen K, Jensen KG, Zhong H, Nielsen SM, Hogg S, Mørk A, Stensbøl TB. Discovery of 1-[2-(2, 4-dimethylphenylsulfanyl) phenyl] piperazine (Lu AA21004): a novel multimodal compound for the treatment of major depressive disorder. Journal of medicinal chemistry. 2011 Apr 12;54(9):3206-3221.

7. Hvenegaard MG, Bang-Andersen B, Pedersen $H$, Jorgensen M, Puschl A, Dalgaard L. Identification of the cytochrome P450 and other enzymes involved in the in vitro oxidative metabolism of a novel antidepressant, Lu AA21004. Drug Metabolism and Disposition. 2012 Jan 1:40(7):1357-1365.

8. Chen G, Lee R, Højer AM, Buchbjerg JK, Serenko M, Zhao Z. Pharmacokinetic drug interactions involving vortioxetine (Lu AA21004), a multimodal antidepressant. Clinical drug investigation. 2013 Oct 1;33(10):727-736.

9. Brintellix (vortioxetine) package insert. Deerfield, Illinois: Takeda Pharmaceuticals America, Inc.; September 2013.

10. Baldwin DS, Loft H, Dragheim M. A randomised, doubleblind, placebo controlled, duloxetine-referenced, fixed-dose study of three dosages of Lu AA21004 in acute treatment of major depressive disorder (MDD). European Neuropsychopharmacology. 2012 Jul 1;22(7):482-491.

11. Katona C, Hansen T, Olsen CK. A randomized, double-blind, placebo-controlled, duloxetine-referenced, fixed-dose study comparing the efficacy and safety of Lu AA21004 in elderly patients with major depressive disorder. International clinical psychopharmacology. 2012 Jul 1;27(4):215-223.

12. McIntyre RS, Lophaven S, Olsen CK. A randomized, doubleblind, placebo-controlled study of vortioxetine on cognitive function in depressed adults. International Journal of Neuropsychopharmacology. 2014 Oct 1;17(10):1557-1567.

13. Häggström L, Nielsen RZ, Poulsen L, Danchenko N. A randomised, double blind, active controlled study of vortioxetine (10-20 mg/day) versus agomelatine $25-50 \mathrm{mg}$ / day) in adults with Major Depressive Disorder with inadequate response to antidepressant treatment. In Poster presented at: 26th Congress of the European College of Neuropsychopharmacology (ECNP) 2013 Oct 5 (pp. 5-9).

14. Alvarez E, Perez V, Dragheim M, Loft H, Artigas F. A doubleblind, randomized, placebo-controlled, active reference study of Lu AA21004 in patients with major depressive disorder. International Journal of Neuropsychopharmacology. 2012 Jun 1;15(5):589-600.

15. Boulenger JP, Loft H, Olsen CK. Efficacy and safety of vortioxetine (Lu AA21004), 15 and $20 \mathrm{mg} /$ day: a randomized, double-blind, placebo-controlled, duloxetine-referenced study in the acute treatment of adult patients with major depressive disorder. International clinical psychopharmacology. 2014 May;29(3):138-149.

16. Henigsberg N, Mahableshwarkar AR, Jacobsen P, Chen $Y$, Thase ME. A randomized, double-blind, placebo-controlled 8-week trial of the efficacy and tolerability of multiple doses of Lu AA21004 in adults with major depressive disorder. The Journal of clinical psychiatry. 2012 Jul;73(7):953-959.

17. Jacobsen PL, Mahableshwarkar AR, Serenko M, Chan S, Trivedi MH. A randomized, double-blind, placebo-controlled study of the efficacy and safety of vortioxetine $10 \mathrm{mg}$ and 20 $\mathrm{mg}$ in adults with major depressive disorder. The Journal of clinical psychiatry. 2015 May 27;76(5):575-582.

18. Jain R, Mahableshwarkar AR, Jacobsen PL, Chen Y, Thase ME. A randomized, double-blind, placebo-controlled 6-wk trial of the efficacy and tolerability of $5 \mathrm{mg}$ vortioxetine in adults with major depressive disorder. International Journal of Neuropsychopharmacology. 2012 Sep 11;16(2):313-321.

19. Mahableshwarkar AR, Jacobsen PL, Chen $Y$, Serenko M, Trivedi MH. A randomized, double-blind, duloxetine-referenced 
study comparing efficacy and tolerability of 2 fixed doses of vortioxetine in the acute treatment of adults with MDD. Psychopharmacology. 2015 Jun 1;232(12):2061-2070.

20. Mahableshwarkar AR, Jacobsen PL, Chen Y. A randomized, double-blind trial of $2.5 \mathrm{mg}$ and $5 \mathrm{mg}$ vortioxetine ( $\mathrm{Lu}$ AA21004) versus placebo for 8 weeks in adults with major depressive disorder. Current medical research and opinion. 2013 Mar 1;29(3):217-226.

21. Mahableshwarkar AR, Jacobsen PL, Serenko M, Chen Y, Trivedi MH. A duloxetine-referenced, fixed dose study comparing efficacy and safety of 2 vortioxetine doses in the acute treatment of adult MDD patients. In American Psychiatric Association (APA) 166th Annual Meeting 2013 May 8.

22. Alvarez E, Perez V, Artigas F. Pharmacology and clinical potential of vortioxetine in the treatment of major depressive disorder. Neuropsychiatric disease and treatment. 2014;10:1297-1307.

23. Boulenger JP, Loft $H$, Florea I. A randomized clinical study of $\mathrm{Lu} \mathrm{AA21004}$ in the prevention of relapse in patients with major depressive disorder. Journal of Psychopharmacology. 2012 Nov;26(11):1408-1416.

24. Baldwin DS, Hansen T, Florea I. Vortioxetine (Lu AA21004) in the long-term open-label treatment of major depressive disorder. Current medical research and opinion. 2012 Oct 1;28(10):1717-1724.
25. Theunissen EL, Street D, Højer AM, Vermeeren A, Van Oers A, Ramaekers JG. A randomized trial on the acute and steady-state effects of a new antidepressant, vortioxetine ( $\mathrm{Lu}$ AA21004), on actual driving and cognition. Clinical Pharmacology \& Therapeutics. 2013 Jun;93(6):493-501.

26. Mahableshwarkar AR, Zajecka J, Jacobson W, Chen Y, Keefe RS. A randomized, placebo-controlled, active-reference, double-blind, flexible-dose study of the efficacy of vortioxetine on cognitive function in major depressive disorder. Neuropsychopharmacology. 2015 Jul;40(8):2025.

27. Baldwin DS, Serenko M, Palo W, Lophaven S, Matz J. The safety and tolerability of vortioxetine (Lu AA21004) in the treatment of adults with major depressive disorder (MDD): a pooled analysis. International Journal of Psychiatry in Clinical Practice. 2013;17:16-17.

28. Citrome L. Vortioxetine for major depressive disorder: a systematic review of the efficacy and safety profile for this newly approved antidepressant-what is the number needed to treat, number needed to harm and likelihood to be helped or harmed? International journal of clinical practice. 2014 Jan;68(1):60-82.

29. Jacobsen PL, Mahableshwarkar AR, Chen Y, Chrones L, Clayton AH. Effect of vortioxetine vs. escitalopram on sexual functioning in adults with well-treated major depressive disorder experiencing SSRI-induced sexual dysfunction. The journal of sexual medicine. 2015 Oct;12(10):2036-2048. 